Piotr ADAMCZEWSKI O.S.P.P.E.

(Roma, Angelicum)

\title{
IL PRESBITERO NELLE LETTERE DI IGNAZIO DI ANTIOCHIA
}

Sono sette le Lettere, accertate dalla critica come autentiche, che scrive Ignazio di Antiochia durante il suo viaggio a Roma: Agli Efesini, Ai Magnesi, Ai Tralliani, Ai Romani, Ai Filadelfiesi, Agli Smirnesi e A Policarpo. Da Smirne scrisse alla comunità di Efeso con il vescovo Onesimo, di Magnesia con Dama e di Tralli con Polibio, che gli avevano mandato apposite rappresentanze. Scrisse anche alla comunità di Roma, pregandola di non intercedere per la grazia. Da Troade scrisse ai fratelli di Filadelfia e di Smirne. Le lettere ci sono tramandate da due manoscritti: Mediceus dell'XI secolo e Colbertinus del X secolo.

Le Lettere di Ignazio toccano il culmine della concezione cristologica espressa dai Padri Apostolici. Sono la voce viva di una esperienza religiosa meditata e sofferta. Ogni lettera incomincia con il saluto di Ignazio Teoforo. Non hanno uno specifico scopo dottrinale. Esse sono occasionali e il loro contenuto è determinato dai bisogni delle Chiese alle quali sono indirizzate. Esse anche hanno un calore spirituale, perché comunicano in una prosa spontanea e semplice gli stati d'animo del santo martire. Inoltre presentano l'unità ecclesiale come un organismo vivo intorno al vescovo nell'armonia dei presbiteri, dei diaconi con tutti i fedeli. Ignazio è il dottore dell'unità. Giornale di viaggio da una parte e diario spirituale dall'altra è il genere letterario di Ignazio. Gli bastano solo pochi riferimenti, per lo più dal Nuovo Testamento, perché il discorso che egli fa sia ricco di sensi biblici nel significato più reale della parola.

Ignazio nelle sue lettere per identificare i presbiteri usa le seguenti parole

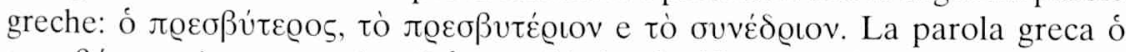

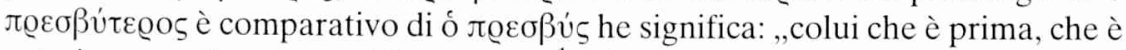
anteriore negli anni, vecchio, canuto" ". Il comparativo significa anche: „più onorato, più venerato, più sacro; i seniori della Chiesa cristiana",2. La parola

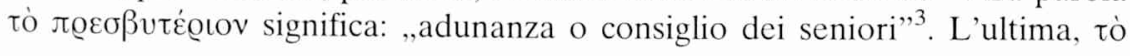

\footnotetext{
Cf. F. Schenkl - F. Brunetti, Dizionario greco - italiano - greco, Genova 1990, 736.

2 Cf. ibidem.

${ }^{3}$ Cf. ibidem.
} 


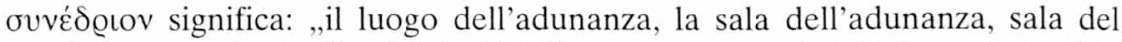
consiglio, un collegio di publici ufficiali con una certa giurisdizione, il sinedrio degli Ebrei” ${ }^{4}$. Nell'esercitazione usiamo due parole italiane: „il presbitero”, che è quasi adeguato a significato greco, ed ,il presbiterio”, che normalmente riguarda una parte della chiesa, ma può esprimere anche il collegio dei presbiteri, e preferiamo l'uso in questo senso.

1. Lettera agli Efesini. La prima lettera intitolata „Alla Chiesa di Efeso” è stata scritta da Ignazio durante la prima tappa del suo viaggio verso Roma, a Smirne. L'autore scrive ai fedeli ed al loro vescovo Onesimo. Tutta la lettera contiene 21 piccoli capitoli più il saluto. Dopo il saluto, che comincia in tutte le lettere nello stesso modo, Ignazio Teoforo passa alle lodi agli Efesini e al loro vescovo. Dopo descrive le diverse questioni: nel II capitolo l'ubbidienza al vescovo e ai presbiteri. Nel III e IV capitolo tocca il tema dell'unità e poi nel $\mathrm{V}$ e VI le caratteristiche personali del vescovo. Dal VII al IX descrive il comportamento nei confronti degli eretici. Poi nel X incoraggia di essere di esempio nelle virtù, e nell' XI di temere il Signore. Nel capitolo XII l'autore esprime la vicinanza del suo martirio e il XIII capitolo lo dedica alla liturgia. Fede e carità sono il tema del capitolo XIV, testimoniare il Cristo del XV, XVI e XVII. Poi l'autore passa al mistero della croce nel capitolo XVIII e all'abolizione della morte. Come ultimi ci sono la promessa di scrivere ancora nel capitolo XX e il congedo nel XXI.

Ignazio nella Lettera agli Efesini scrive direttamente sul tema dei presbiteri, o meglio del presbiterio, tre volte, nei capitoli II, IV e XX:

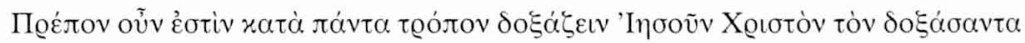

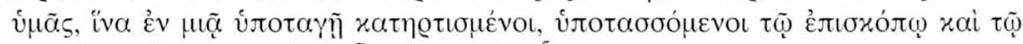

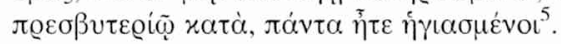

In questo frammento Ignazio riferendosi all'obbedienza e alla sottomissione, subito dopo aver parlato del vescovo parla anche del presbiterio. L'unità che deve essere nella Chiesa ha come punto di arrivo il vescovo con il suo presbiterio composto dai presbiteri. Ignazio sottolinea nelle sue lettere l'unità e la spiega. Non si può dire che i poteri dei presbiteri siano uguali a quelli del vescovo, poiché quest'ultimo è il garante dell'unità, ma il significato del presbiterio e il suo ruolo vengono da quest'unità con il vescovo. Il posto occupato dai presbiteri è subordinato al vescovo e l'obbedienza e la sottomissione loro porta ai fedeli la santificazione che glorifica Gesù:

${ }^{4}$ Cf. ibidem, 837.

5 Epistula ad Ephesios 2, 2, SCh 10, 58: „Bisogna glorificare in ogni modo Gesù Cristo che ha glorificato voi, perché riuniti in una stessa obbedienza e sottomessi al vescovo e ai presbiteri siate santificati in ogni cosa". 


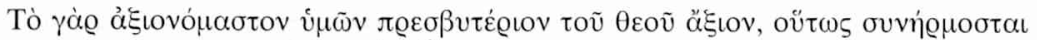

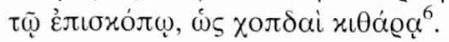

Su questo passaggio del capitolo IV Ignazio descrive l'unione del collegio presbiterale con il vescovo usando il paragone che viene dalla musica. L'unione deve essere come l'armonia delle corde della cetra. Il frammento precedente dichiara che procedere d'accordo con la mente del vescovo è conveniente ai fedeli. Il presbiterio efesino è l'esempio dell'unione con il vescovo. E' composto non da una persona, ma dai molti anziani e nel loro procedimento tutti seguono la mente, la linea del vescovo per non creare nessuna disarmonia nella Chiesa. Quest'esempio è molto forte, perché Ignazio usando il paragone che viene dalla musica rafforza il significato dell'unione del presbiterio con il vescovo, dove non c'è posto per la disarmonia. Se ne possono dedurre le qualità che appartengono ai presbiteri. Bisogna conoscere bene il posto, l'età e tutto ciò che caratterizza l'ambiente per descrivere in modo sufficiente i particolarismi della vita quotidiana e le proprietà di questa gente. Ma sicuramente possiamo affermare che per soddisfare abbastanza quest'unione tra il vescovo e i presbiteri occorrono persone mature, oneste, che hanno ben capito l'insegnamento di Gesù e riescono a realizzarlo nella vita, in modo da fare veramente unità con il vescovo nella mente, nell'insegnamento, nel procedimento e nel comportamento, diventando un buon esempio per gli altri. Per Ignazio quest'unità fa il vero coro che canta ad una sola voce a Dio per partecipare sempre in Lui.

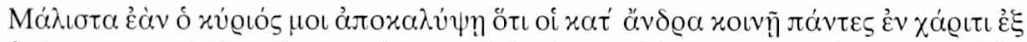

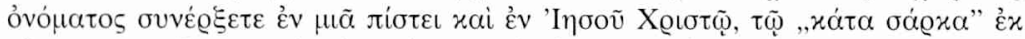

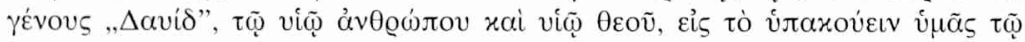

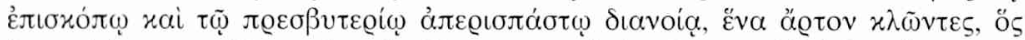

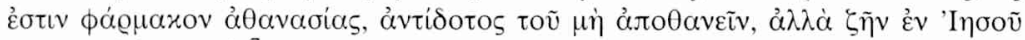

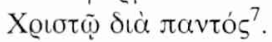

Nel capitolo XX torna il discorso sul presbiterio e sul vescovo, in rapporto all'obbedienza dei fedeli. Nel contesto dell'unità intorno ad un altare e all'unico pane, Ignazio chiama tutti fedeli della Chiesa efesina ad ininterrotta concordia nell'obbedienza al vescovo ed ai presbiteri. Nuovamente il presbiterio appare come un gruppo che partecipa all'eucaristia come più vicino al vescovo. L'unione dei fedeli e del vescovo con il presbiterio in una sola fede per nutrirsi del pane

${ }^{6}$ Ibidem, 4, 1, SCh 10, 60: „Il vostro presbiterato ben reputato degno di Dio è molto unito al vescovo come la corda alla cetra".

${ }^{7}$ Ibidem, 20, 2, SCh 10, 76: ,Sovrattutto se il Signore mi rivelerà che ognuno e tutti insieme nella grazia che viene dal suo nome vi riunite in una sola fede e in Gesù Cristo del seme di David figlio dell'uomo e di Dio per ubbidire al vescovo e ai presbiteri in una concordia stabile spezzando l'unico pane che è rimedio di immortalità, antidoto per non morire, ma per vivere sempre in Gesù Cristo". 
diventa rimedio per vivere in Gesù. Certamente i presbiteri, pur essendo anche i fedeli della Chiesa, sono più vicini al vescovo e anche si distinguono da essi: ciò significa che il loro ruolo è più ampio di quello di semplici consiglieri. Questa posizione è rafforzata attraverso lo scopo a cui conduca l'eucaristia, cioè attraverso l'immortalità, la vita eterna, il vivere in Cristo.

2. Lettera ai Magnesi. La lettera di Ignazio alla Chiesa di Magnesia, dove presiede il vescovo Dama, è una delle quattro, scritte da Ignazio durante la prima tappa del suo viaggio. Ignazio scrive anche questa lettera mentre si trovava a Smirne. Egli ha incontrato il vescovo di Magnesia con due presbiteri ed il diacono e racconta quest'incontro nei primi capitoli della sua lettera. Tutta la lettera consta di 15 capitoli più il saluto. Il I capitolo contiene l'insegnamento dell'unione col Cristo, i due seguenti trattano della sottomissione al vescovo, poi usando l'immagine delle due monete spiega il destino umano: la morte o la vita eterne. La concordia occupa il capitolo VI, l'immagine del tempio unico di Dio è simbolo dell'unità nel VII capitolo. Nell' VIII l'autore spiega il significato di ricevere la grazia, poi nel IX spiega l'importanza della domenica. Il tipo biblico del nuovo lievito è usato e dichiarato nel X capitolo. L'autore esprime nell' XI capitolo la sua piena e ferma adesione a Cristo, nel seguente afferma sua uguaglianza con gli altri. Nel terzultimo capitolo l'autore incoraggia ad osservare i precetti del Signore e degli apostoli; in fine Ignazio passa al congedo.

In questa lettera sei passi trattano direttamente dei presbiteri e del presbiterio: nei capitoli II, III, VI e VII dei presbiteri e nei capitoli II e XIII del presbiterio.

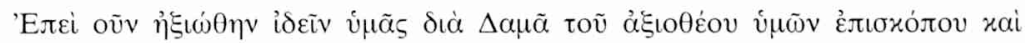

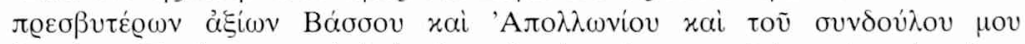

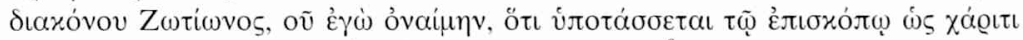

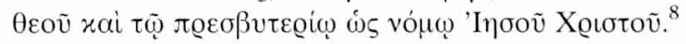

Per la prima volta nelle sue lettere Ignazio non parla solo del presbiterio, del consiglio degli anziani, ma direttamente dei presbiteri. Ricorda due di loro: Basso ed Apollonio, egli li ha incontrati con il loro vescovo e il diacono Zootione. Essi sono la buona e degna rappresentazione della loro Chiesa in Magnesia. Sono tutte persone che s'identificano ufficialmente con la Chiesa particolare: il vescovo, i presbiteri ed i diaconi. Esiste un ordine che permette di distinguere tre diverse unità fra i rappresentanti ufficiali della Chiesa, ma le lettere di Ignazio non dicono niente della caratteristica essenziale della diffe-

${ }^{8}$ Epistula ad Magnesios 2, 2, SCh 10, 80: „Ho avuto l'onore di vedervi in Dama, vostro vescovo degno di Dio, nei degni presbiteri Basso ed Apollonio e nel diacono Zootine, mio conservo, della cui presenza mi auguro sempre gioire. Egli è sottomesso al vescovo come alla grazia di Dio e al presbitero come alla legge di Gesù Cristo". 
renza fra di loro. Il presbiterio nel suo carattere è più vicino al vescovo ed è più alto nella dignità rispetto ai diaconi. La posizione dei diaconi ormai era fissata negli Atti degli Apostoli e Ignazio, definendosi il „conservo” come il diacono, fa quest'atto per umiltà, ma afferma che le posizioni dei presbiteri e dei diaconi non erano uguali e anche gli impegni erano diversi. I presbiteri sono uniti con il vescovo nella liturgia ed anche nell'ordine della vita della Chiesa.

Il passaggio che segue definisce con chiarezza che il diacono è sottomesso al vescovo e ai presbiteri. Ci porta anche un paragone: il vescovo e la grazia di Dio, il presbiterio e la legge di Gesù Cristo. Questo passo in primo luogo esprime la natura della differenza tra il vescovo e il presbitero. Il ruolo del vescovo è più libero e più ampio come la grazia, il presbitero ha per sua attività pastorale un territorio ben definito come la legge. Chiaramente possiamo dire che l'obbedienza al vescovo e al presbiterio nella Chiesa riguardo alla fede, la moralità, la liturgia è ben definita. Dato che la natura della grazia è diversa della legge, quali saranno le proprietà della grazia in confronto alle qualità della legge? La risposta concreta non è facile. Sicuramente possiamo affermare che i presbiteri nella loro vita ecclesiale hanno meno potestà del vescovo in confronto ai fedeli. Sembra che la potestà dei presbiteri riguarda il livello quotidiano e intero della Chiesa, applicando le usanze e i costumi già fatti; i vescovi hanno i poteri più ampi e con la grazia possono superare la legge non rompendola.

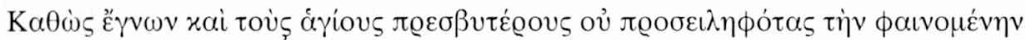

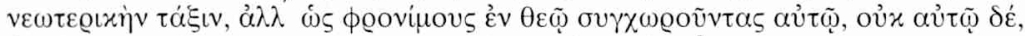

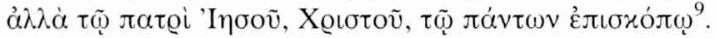

Tutto il terzo capitolo parla della sottomissione al vescovo. Nel passaggio citato Ignazio loda i presbiteri che sono sottomessi al giovane vescovo non sottovalutando sua giovinezza. Il presbiterio, essendo più vicino al vescovo, può influenzarla. Se resistono a questa tentazione, come afferma Ignazio, sono persone ben scelte che non guardano il proprio vantaggio ma il vero bene della Chiesa e dei fedeli, che cercano la vera unità intorno al vescovo, che rappresenta secondo Ignazio „il Padre di Gesù che è il vescovo di tutti”. I presbiteri sono saggi in Dio, cioè con il loro comportamento manifestano che la provvidenza dà senso a tutto.

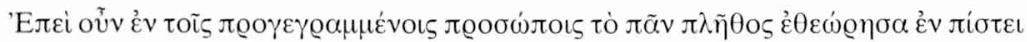

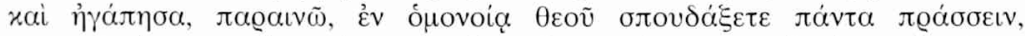

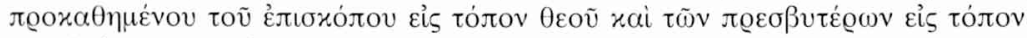

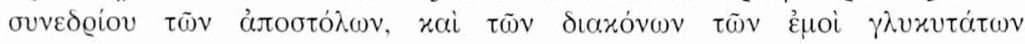

9 Ibidem, 3, 1, SCh 10, 82: „In realtà ho saputo che i vostri santi presbiteri non hanno abusato della giovinezza evidente in lui, ma saggi in Dio sono sottomessi a lui, non a lui, ma al Padre di Gesù Cristo che è il vescovo di tutti". 


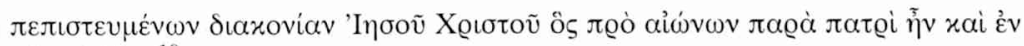
$\tau \varepsilon \dot{\lambda} \varepsilon \varepsilon\left\llcorner\dot{\varepsilon} \phi \dot{\alpha} \eta^{10}\right.$.

Vediamo il nuovo paragone. Il vescovo con i presbiteri e i diaconi guida la comunità ecclesiastica. Il vescovo è al posto di Dio, i presbiteri al posto del collegio apostolico. Tutti essi con i diaconi svolgono il servizio di Gesù Cristo. Il presbitero è un servo che svolge il suo servizio come apostolo. Il vescovo è per la comunità, e come Gesù per i discepoli e gli apostoli. Essi portano la Buona Novella alla gente. Servono il popolo là, dove il vescovo non può essere presente. Essi possono servire così perché sono nell'unità col vescovo e rappresentano lui. Di nuovo l'autore afferma la vicinanza tra il vescovo e il presbiterio, ma aggiunge che i presbiteri prolungano l'azione del vescovo, l'attività pastorale dei servi del popolo cristiano.

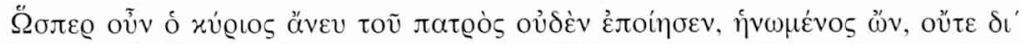

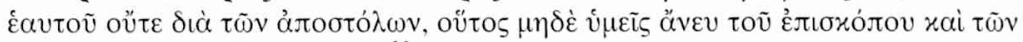

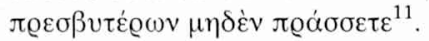

La piena guida della Chiesa particolare è il vescovo con il suo presbiterio. Ignazio ancora una volta pone l'accento sull'unità fra il vescovo ed il presbiterio. Questa cooperazione presbiterale diventa importante per i fedeli, che devono pienamente collaborare con il presbiterio, seguendo Gesù nella sua sottomissione al Padre. Pure l'autore accenna al raggio d'azione dei presbiteri: sotto la presidenza del vescovo essi dirigono la Chiesa verso il Padre e servono i cristiani.

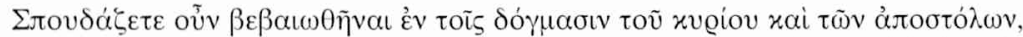

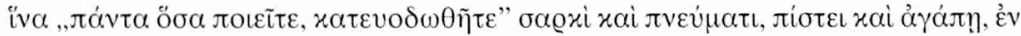

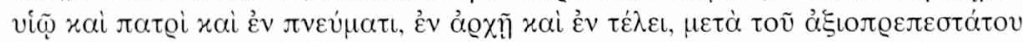

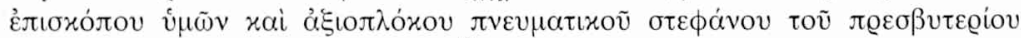

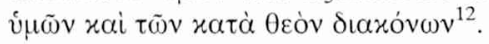

Nell'ultimo passaggio della lettera ai Magnesi che parla dei presbiteri il collegio presbiterale è chiamato „la preziosa corona spirituale”. Che cosa in

${ }^{10}$ Ibidem, 6, 1, SCh 10, 82-84: „Poiché nelle persone nominate sopra ho visto e amato tutta la comunità vi prego di essere solleciti a compiere ogni cosa nella concordia di Dio e dei presbiteri. Con la guida del vescovo al posto di Dio, e dei presbiteri al posto del collegio apostolico e dei diaconi a me carissimi che svolgono il servizio di Gesù Cristo che prima dei secoli era presso il Padre e alla fine si è rivelato".

${ }^{11}$ Ibidem, 7, 1, SCh 10, 84: „Come il Signore nulla fece senza il Padre col quale è uno, né da solo né con gli apostoli, così voi nulla fate senza vescovo e i presbiteri”.

12 Ibidem, 13, 1, SCh 10, 90: „Cercate di tenervi ben saldi nei precetti del Signore e degli apostoli perché vi riesca bene tutto quanto fate nella carne e nello spirito, nella fede e nella carità, nel Figlio, nel Padre e nello Spirito, al principio e alla fine, con il vostro vescovo che è tanto degno e con la preziosa corona spirituale dei vostri presbiteri e dei diaconi secondo Dio". 
particolare rappresenta il presbiterio, tanto da essere chiamato la corona, rafforzato dai due aggettivi? Sola la corona normalmente esprime la potestà reale e la ricchezza del proprietario, essendo anche un elemento decorativo. Dio è il re di tutti noi; i presbiteri, essendo una corona, sono una parte della Chiesa che per la sua sostanza e l'attività orna tutta la Chiesa che appartiene a Dio. Questa parte, cioè i presbiteri, compiono la vocazione della Chiesa portando la salvezza che viene da Dio alle genti attraverso il suo servizio, servizio dei vescovi, dei presbiteri e dei diaconi. Tutto quel processo, al quale partecipano i presbiteri, diventa una corona che simboleggia il compimento della promessa fatta da Dio, che si realizza nel servizio proveniente da Dio, il servizio del presbiterio e dei diaconi. L'importanza del presbiterio riguarda la sfera spirituale della Chiesa cioè l'insegnamento acquistato da Gesù. La corona è preziosa, è raffinata; secondo Ignazio, è anche importante per i presbiteri che la creano. Ignazio aggiunge: , dei vostri presbiteri”, ciò vuol dire che i presbiteri sono parte essenziale della comunità, scelti da essa.

3. Lettera ai Tralliani. La lettera indirizzata alla Chiesa in Tralli è la terza delle quattro, scritte da Ignazio quando si è fermato in Smirne. Il vescovo di Tralli, Polibio, è venuto a Smirne con due altri a salutarli. Ignazio scrive poi una lettera composta da 13 capitoli più il saluto. Come in tutte le altre, Ignazio inizia con le parole: Ignazio, Teoforo. Dopo il saluto, nel primo capitolo l'autore ricorda l'incontro con il vescovo Polibio e l'impressione avuta fin dall'inizio. Dopo passa alla sottomissione al vescovo che è per la comunità come Gesù. Il capitolo seguente contiene la meditazione di Ignazio che riguarda la Chiesa che non può esistere senza i diaconi, i presbiteri e il vescovo. Nel capitolo IV e V l'autore esprime la sua volontà di diventare vero discepolo di Cristo, e anche tocca la realtà del cielo. Il VI capitolo contiene la preoccupazione d'avere la pura fede. Dopo, nel capitolo VII, Ignazio usando l'immagine del santuario chiama i fedeli ad operare nell'unità, e nel capitolo seguente a ricrearsi nella fede e nella carità. Nel capitolo IX Ignazio afferma che senza Gesù, vero Cristo ed uomo, non abbiamo la vera vita. Poi spiega perché è incatenato e va a morire. L'immagine della pianta del Padre nell' XI capitolo serve all'autore per chiarire chi dà il frutto buono. Gli ultimi due capitoli, XII e XIII, contengono il congedo di Ignazio.

I passi che comprendono i testi riguardanti i sacerdoti nella lettera $\mathrm{Ai}$ Tralliani sono nei capitoli II, III, VII, XII e XIII.

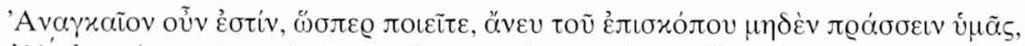

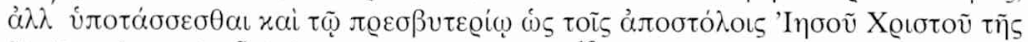

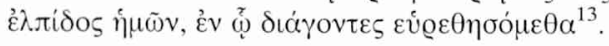

${ }^{13}$ Epistula ad Trallianos 2, 2, SCh 10, 96: „E' necessario, come già fate, non operare nulla senza il vescovo, ma sottomettervi anche ai presbiteri come agli apostoli di Gesù Cristo speranza nostra, e in lui vivendo ci ritroveremo". 
Di nuovo il collegio presbiterale è paragonato agli apostoli. Il capo della Chiesa particolare è il vescovo, ma la sua azione è prolungata dai presbiteri che, autorizzati, operano e servono come collaboratori del vescovo, ma anche edificano un gruppo che serve al vescovo come ausilio. Già abbiamo parlato del presbiterio che è stabilito intorno al vescovo. Chiaramente tutto questo discorso ci guida all'unità. In quest'unità il presbiterio occupa un suo proprio posto. Non conviene omettere i presbiteri operando senza di loro. Nel passo citato sembra che Ignazio voglia più decisamente indicare il posto del presbiterio che con il vescovo guida la comunità cristiana della Chiesa. La sottomissione ai presbiteri, che sono nell'unità con il vescovo, porta la sicurezza di operare secondo l'insegnamento della Chiesa.

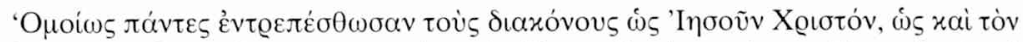

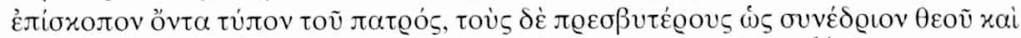

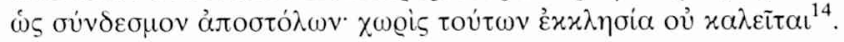

Ignazio decisamente indica che la Chiesa ha bisogno dei vescovi, dei presbiteri e dei diaconi. Senza di loro non esiste la Chiesa. Essi formano un organismo importante, senza di cui la Chiesa non può continuare la sua essenziale missione. In questo frammento della lettera ai Tralliani Ignazio al posto di „sottomissione” usa il „rispetto" per indicare ai fedeli i loro pastori. La sfumatura del „rispetto" è diversa dalla sottomissione. Il rispetto esprime più di una semplice accettazione, nel senso di riverenza dell'autorità. L'autore aggiunge un nuovo paragone che riguarda i presbiteri. Essi sono come il collegio degli apostoli e come il sinedrio di Dio. Questo paragone con il più alto consiglio ebreo sviluppa la conoscenza del ruolo dei presbiteri. Il sinedrio era per gli Ebrei la più alta autorità religiosa. Per la sua scienza il sinedrio istruiva e dava le spiegazioni alla gente nelle difficoltà di capire la legge. Tra i suoi membri veniva scelto il sommo sacerdote, che poteva entrare nel tempio di Gerusalemme, una volta all'anno, nel santo dei santi. Il consiglio dei presbiteri compie un ruolo simile. E' composto dagli anziani, saggi che servono i fedeli con la loro saggezza e l'esperienza. Per i loro studi approfondiscono la fede ed avvicinano i fedeli a Dio compiendo la missione della Chiesa. Uno di loro a causa delle sue virtù, della salda fede, diventa il vescovo. La missione della Chiesa si esprime anche nel compiere il precetto di Gesù di spezzare il pane, cioè nel celebrare l'eucaristia. I presbiteri partecipano nell'eucaristia accompagnando il vescovo che presiede. Ma la loro posizione è anzitutto collegata con il servizio al vescovo ed ai fedeli.

${ }^{14}$ Ibidem, 3, 1, SCh 10, 96: „Similmente tutti rispettino i diaconi come Gesù Cristo, come anche il vescovo che è l'immagine del Padre, i presbiteri come il sinedrio di Dio e come il collegio degli apostoli. Senza di loro non c'è Chiesa". 


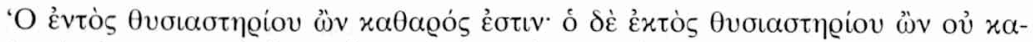

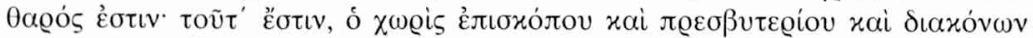

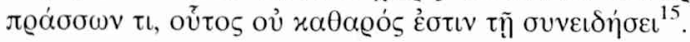

Ancora una volta Ignazio nelle sue lettere esprime l'unità, la struttura della Chiesa ed il posto che occupano i presbiteri in questa struttura. Anche il fatto che ,partecipano in tutto” è importante per la Chiesa, cioè nella missione della Chiesa. Questo passo di nuovo afferma che il presbiterio è sostanziale nella Chiesa. L'autore ripeterà questa formulazione ancora perché fa parte della sua visione della Chiesa unita intorno al vescovo.

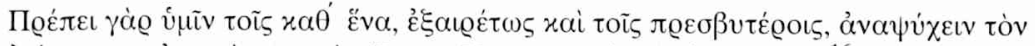

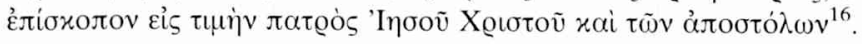

I presbiteri, come già abbiamo notato, formano un collegio dei collaboratori del vescovo. La loro attività si svolge anzitutto nel servizio ai fedeli, ma vediamo anche che esiste una parte delle loro attività che riguarda il vescovo. Tutti devono „confortare” il vescovo, in particolare i presbiteri. In quale senso si esprime l'azione di confortare? Lo esprime meglio il verbo „rafforzare”. Possiamo distinguere due livelli: naturale o umano e di fede. Il collegio presbiterale aiuta il suo vescovo come noi aiutiamo gli amici, preoccupandosi di loro. Ma anche aiuta il vescovo con il proprio consiglio. E questo è giusto. Il vescovo serve alla comunità ed essa fa lo stesso per lui, soprattutto quelli che sono più vicino al vescovo, appunto il collegio presbiterale.

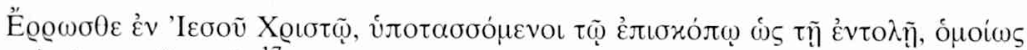

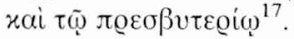

Ignazio nell'ultimo capitolo della lettera ai Tralliani ricorda ai fedeli della Chiesa in Tralli che devono essere forti nella fede sottomettendosi al vescovo ed ai presbiteri. La fede forte e salda deve essere riconosciuta dal vescovo e dai presbiteri come pastori che nell'unità, raccolti intorno al vescovo, guidano la Chiesa. Essere in Cristo significa essere nell'unità con il vescovo e con i presbiteri per partecipare pienamente nella Chiesa di Gesù Cristo.

4. Lettera ai Romani. La lettera di Ignazio, intitolata „Ai Romani”, è stata scritta anch'essa a Smirne, durante la sua presenza. Ha un carattere un po' diverso, perché Ignazio scrive alla comunità che raggiunge per subire il marti-

15 Ibidem, 7, 2, SCh 10, 100: „Ciò significa che chiunque operi separatamente dal vescovo, dal presbitero e dai diaconi, non è puro nella coscienza”.

${ }^{16}$ Ibidem, 12, 2, SCh 10, 102: „Conviene che ciascuno di voi, e in particolare i presbiteri, conforti il vescovo nella gloria del Padre di Gesù Cristo e degli apostoli”.

17 Ibidem, 13, 2, SCh 10, 102: „Siate forti in Gesù Cristo, sottomessi al vescovo, come al comandamento e ai presbiteri". 
rio. Non la conosce personalmente, e questo implica che doveva scrivere in altro modo. Tutta la lettera contiene 10 capitoli. Il tema generale riguarda il martirio, che dovrebbe unire con Cristo come il vero discepolo. Ignazio in questa lettera non scrive niente che tocca direttamente i presbiteri. Si concentra su altri temi come abbiamo detto prima. Dopo il saluto, nel I capitolo, esprime la sua volontà di vedere la Chiesa romana e di compiere il suo martirio per diventare un vero discepolo di Cristo. Il capitolo seguente esprime la sua preparazione per ricevere il martirio. Il III capitolo contiene l'incitamento di perseverare nell'insegnamento di Gesù, nonostante l'odio del mondo verso il cristianesimo. Nel IV capitolo l'autore paragona se stesso con il frumento che deve morire, e che deve essere macinato per diventare pane puro. Poi Ignazio di nuovo parla della sua volontà di diventare discepolo di Cristo attraverso il martirio, di raggiungere Cristo in questo modo. Similmente, nel capitolo VI, Ignazio esorta ad imitare la passione di Gesù. Nel VII capitolo dà consigli di resistere al principe di questo mondo e ripete la sua volontà di martirio. Nel terzultimo capitolo Ignazio chiede alla Chiesa di Roma di ricevere la sua lettera, come scritta secondo la mente di Dio. I due ultimi capitoli contengono il congedo.

5. Lettera ai Filadelfiesi. La lettera alla Chiesa in Filadelfia è la prima delle tre Lettere di Ignazio scritte durante la seconda tappa del suo viaggio verso Roma. Ignazio, partito da Smirne viene a Troade, dove scrive le nuove lettere. Tutta la lettera contiene 11 capitoli. Come tutte le altre, essa inizia con il saluto: „Ignazio, Teoforo", dopo di quale l'autore spiega in modo generale l'impegno del vescovo, basandosi sull'esempio del loro vescovo. Poi consiglia di sfuggire la faziosità e le dottrine perverse. Usando l'immagine delle erbe cattive suggerisce nel capitolo III di stare lontano da quelli che non sono nell'unità con la Chiesa, con il vescovo e nel capitolo seguente, partendo dall'unica carne e sangue di Gesù chiama i fedeli a riunirsi in una sola eucaristia intorno al vescovo. Nel V capitolo ricorda l'esempio dei profeti che prima hanno annunziato Gesù ed il vangelo. Dopo nuovamente chiede di essere attenti alle male arti e agli inganni del mondo. Nel VII capitolo ricorda che essi devono preoccuparsi del proprio corpo come del tempio di Dio. Poi incoraggia a seguire Gesù e la vera dottrina, senza la fazione, l'ira, lo spirito di contesa. Nel capitolo IX l'autore scrive del compimento nel vangelo dell'incorruttibilità. Nel capitolo seguente Ignazio esprime la sua gioia a causa della pace in Antiochia, della cessazione delle persecuzioni. L'ultimo capitolo contiene il congedo.

Ignazio nella lettera ai Filadelfiesi si riferisce ai presbiteri sei volte: nel saluto e nei capitoli IV, V, VII, VIII e X.

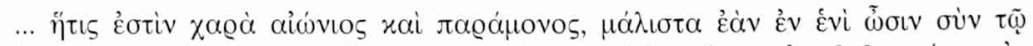

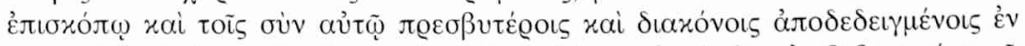

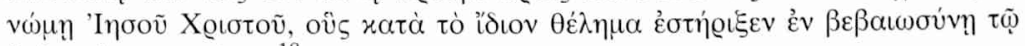

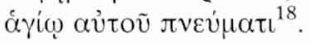


Il primo passo tocca anzitutto l'unità della Chiesa. Possiamo notare due nuovi aspetti che riguardano ai presbiteri: sono scelti, secondo la volontà di Gesù, sono confermati e fortificati con lo Spirito Santo. Il primo aspetto esprime la vocazione, oppure la scelta che viene da Gesù. Per diventare presbitero occorre qualche particolare processo per essere sicuri che il candidato è degno di essere presbitero. Poi i vescovi, i presbiteri e i diaconi ricevono lo Spirito Santo, che conferma la loro vocazione e la fortifica. Possiamo affermare che per dirigere e servire la Chiesa i presbiteri ricevono doni particolari che permettono di compiere meglio la loro vocazione.

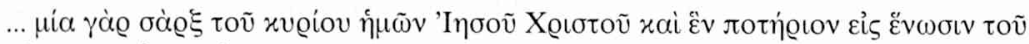

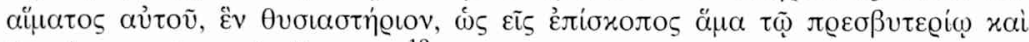

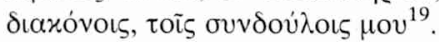

Si tratta di un altro passaggio che riguarda soprattutto l'unità della Chiesa e l'eucaristia. La Chiesa, nella sua funzione liturgica è composta da un solo vescovo, i presbiteri, i diaconi ed i fedeli. E' paragonata all'unica carne di Cristo, un calice del sangue, un altare. Queste realtà riguardano la sostanza della Chiesa e della sua missione. Il presbiterio è sostanzialmente incarnato nella complessa struttura della Chiesa: ciò Ignazio dimostra tante volte. Occupa il posto ministeriale, cioè serve alla comunità della Chiesa e con i diaconi ed i fedeli, intorno al vescovo, crea l'unità della Chiesa particolare.

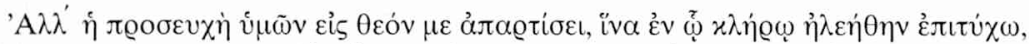

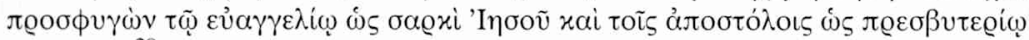
$\dot{\varepsilon} x \lambda \lambda \eta \sigma \alpha \varsigma^{20}$.

Come prima, Ignazio vede qui la similitudine tra gli apostoli e il presbiterio della Chiesa particolare. Possiamo dedurre che il presbiterio, i presbiteri seguendo il collegio degli apostoli, aiutano tutti coloro che cercano rifugio nelle difficoltà e nei dubbi della fede. Gli apostoli, accanto agli altri impegni, hanno incoraggiato i fedeli, hanno edificato e approfondito loro fede. Similmente devono fare i presbiteri. Il vescovo è solo, ma essi sono più diffusi e possono rafforzare i fedeli nelle difficoltà attraverso il loro servizio. Per il loro l'insegnamento e l'esempio, gli apostoli sono diventati i testimoni di Cristo e della

${ }^{18}$ Epistula ad Philadelphenses. Tit., SCh 10, 120: „Essa è il mio eterno e continuo giubilo specialmente se «i fedeli» sono in uno col vescovo e con i suoi presbiteri e con i diaconi scelti nella mente di Gesù Cristo che, secondo la sua volontà, ha confermati col suo Santo Spirito".

19 Ibidem, 4, 1, SCh 10, 122: „Una è la carne di nostro Signore Gesù Cristo e uno il calice nell'unità del suo sangue, uno è l'altare come uno solo il vescovo con il presbiterato e i diaconi, miei conservi".

${ }^{20}$ Ibidem, 5, 1, SCh 10, 124: „Ma la vostra preghiera in Dio mi perfezionerà per raggiungere misericordiosamente l'eredità, rifugiandomi nel vangelo come nella carne di Gesù e negli apostoli, come nel presbiterato della Chiesa". 
fede. Nella testimonianza si svolge anche il ruolo dei presbiteri. Essi danno l'esempio alla gente, perché, come ormai abbiamo detto, i presbiteri devono esser le persone mature, incorporate nella missione ricevuta dal Cristo.

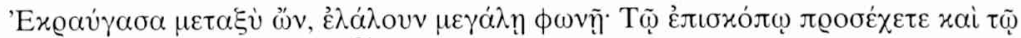

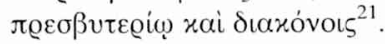

Ignazio senza dubbio è il dottore dell'unità. Con voce alta chiama i fedeli ad essere sottomessi al vescovo, ai presbiteri e ai diaconi. Vediamo di nuovo la struttura della Chiesa particolare composta dal vescovo, dai presbiteri e dai diaconi. Ignazio rafforza la sua richiesta gridando ad alta voce, una voce che viene da Dio.

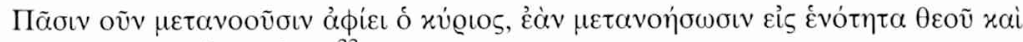

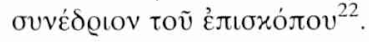

Questo frammento parla del perdono, e spiega anche alcuni aspetti della Chiesa particolare. La sorgente del perdono è solo Dio, ma chi cerca il perdono si deve pentire per Dio e per il sinedrio del vescovo. Il sinedrio rappresenta l'azione di Dio nel mondo materiale. Per il ,sinedrio del vescovo" intendiamo il vescovo che ne fa parte, ed il presbiterio. Sicuramente in questo processo il vescovo rappresenta di più l'autorità della Chiesa, ma per evitare dubbi e rafforzare la posizione del vescovo, questo ultimo non si presenta da solo, ma con il collegio presbiterale che serve il suo vescovo. Vediamo che in questa situazione i diaconi sono esclusi e il loro impegno è diverso. Ma i presbiteri rimangono come i collaboratori più vicini del vescovo, ed essendo uno con lui rappresentano insieme l'autorità della Chiesa nel processo dl perdono.

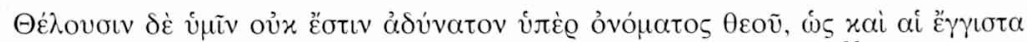

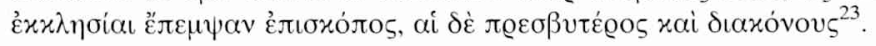

Questo è un altro passaggio che riguarda i presbiteri. Ignazio, dopo aver ricevuto l'annunzio che le persecuzioni sono cessate in Antiochia, vuole che la Chiesa di Filadelfia elegga un diacono e lo mandi in Siria per portare i saluti, i rallegramenti e gli auguri a causa della pace alla sua propria Chiesa antiochena. L'autore scrive che questo servizio onorevole lo compiono di solito i diaconi, i presbiteri ma anche i vescovi. Noi notiamo come novità l'impegno dei presbiteri: oltre al ministero nella propria Chiesa, essi portano anche il loro

${ }^{21}$ Ibidem, 7, 1, SCh 10, 126: „Quando ero in mezzo a voi gridai e a voce alta: state uniti al vescovo, ai presbiteri e ai diaconi”.

${ }^{22}$ Ibidem, 8, 1, SCh 10, 126: ,Il Signore perdona a chi si pente, se si pente per l'unità di Dio e il sinedrio del vescovo".

${ }^{23}$ Ibidem, 10, 1, SCh 10, 130: „A voi che lo volete non sarà impossibile per il nome di Dio come anche le Chiese vicine mandarono i vescovi, oltre i presbiteri e i diaconi”. 
servizio alle altre Chiese. Diventano con i diaconi un mezzo ufficiale delle comunicazioni tra le Chiese particolari e messaggeri del vescovo.

6. Lettera agli Smirnesi. La sesta lettera indirizzata alla Chiesa in Smirne è stata scritta da Ignazio, come la precedente, a Troade, dove si fermava viaggiando a Roma. La lettera contiene il saluto più 13 capitoli. Nel primo capitolo si parla di Gesù, vero uomo che realmente era nato dalla vergine e morì sulla croce per gli uomini. Il capitolo seguente è dedicato alla realtà delle sofferenze e della risurrezione di Cristo. Poi Ignazio testimonia la risurrezione nella carne di Gesù. Essendo consapevole della realtà carnale di Gesù, nel IV capitolo chiede ai fedeli di sopportare tutto in Cristo. La passione del Signore è la risurrezione dei fedeli, e questa posizione difende Ignazio nel capitolo $\mathrm{V}$, poi nel seguente spiega ai lettori il significato della fede e della carità nel comportamento cristiano. Critica la faziosità, avvisa di stare lontano da quelli che contestano l'eucaristia come vera carne di Gesù. Ignazio dedica il capitolo VIII al comando di seguire il vescovo con il suo presbiterio. Riconoscere il vescovo e onorarlo è giusto; di questo parla nel capitolo IX. Ignazio, ringraziando la comunità di Smirne, offre la sua vita e le sue catene. Nell' XI capitolo, prima del congedo e come nella lettera Ai Filadelfiesi, Ignazio chiede alla comunità di Smirne di eleggere uno per andare ad Antiochia a congratularsi con la sua Chiesa per aver riacquistato la pace. Gli ultimi due capitoli, il XII e XIII, contengono il congedo.

La lettera alla Chiesa di Smirne contiene due passi che parlano direttamente dei presbiteri, nei capitoli VIII e XII.

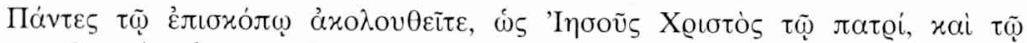

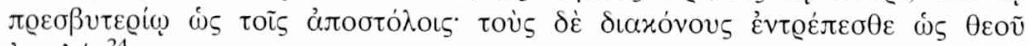

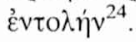

In questo passaggio della lettera agli Smirnesi Ignazio porta ai fedeli l'esempio di Gesù che seguiva Dio Padre, compiendo la sua volontà. Anche i fedeli devono comportarsi così verso il loro vescovo ed i presbiteri, seguendo loro come gli apostoli. Sembra che nel pensiero di Ignazio il vescovo con il suo presbiterio rappresenta l'autorità, che è uguale all'autorità degli apostoli. Possiamo supporre che in qualche modo il presbiterio, come consiglio, sostituisce l'esperienza diretta della conoscenza personale di Gesù, che gli apostoli avevano. Imparavano direttamente da Lui e come maestri della fede hanno esperienze più profonde. Tutto questo non mette i vescovi sul livello più basso degli apostoli; niente nelle Lettere di Ignazio suggerisce questo, ma per avere più sicurezza nella fede i vescovi sono aiutati dai presbiteri, dal collegio presbite-

${ }^{24}$ Epistula ad Smyrnaeos, 8, 1, SCh 10, 138: „Come Gesù Cristo segue il Padre, seguite tutti il vescovo e i presbiteri come gli apostoli; venerate i diaconi come la legge di Dio". 
rale. Gli apostoli come primi hanno presieduto le Chiese particolari, dopo di loro vengono i vescovi, consigliati dai presbiteri. Di nuovo possiamo dedurre quale ruolo compiono in questo caso i presbiteri. Essi sono i consiglieri del vescovo che si preoccupano della fede, della comunità e sono un gruppo che è più vicino al vescovo. Sappiamo anche da altri passi che i presbiteri prolungano l'azione pastorale del vescovo. La Chiesa particolare è condotta dal vescovo con i suoi presbiteri.

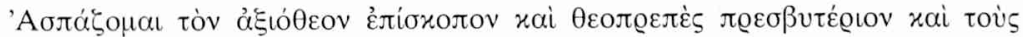
'

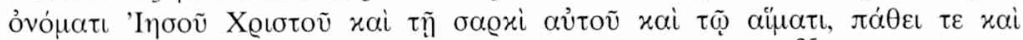

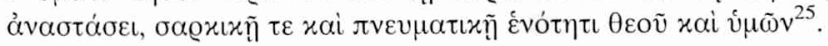

Questo secondo ed ultimo passo relativo ai presbiteri nella lettera Agli Smirnesi non ha tanta importanza. Ignazio nel congedo elenca prima il vescovo, poi i presbiteri e i diaconi. Al vescovo ed ai presbiteri aggiunge gli avverbi. Praticamente entrambi significano: degno di Dio. Se ci rendiamo conto che il vescovo è scelto dal collegio presbiterale per condurre la Chiesa particolare, non ci si stupisce della somiglianza tra le definizioni aggiunte.

7. Lettera a Policarpo. La settima lettera di Ignazio, è - a differenza delle altreá- indirizzata solo al vescovo di Smirne, Policarpo. E' stata scritta, come le due precedenti, in Troade. E anch'essa, come tutte altre, comincia con il saluto: „Ignazio, Teoforo”. Essa contiene 8 capitoli. Nel I capitolo Ignazio loda la pietà di Policarpo, ben fondata sull'insegnamento di Gesù ed anche lo incoraggia a resistere bene. Usando la parabola evangelica del serpente e della colomba, gli consiglia d'essere prudente come il primo e semplice come l'altro animale, nella sua vita di vescovo. Il III capitolo porta l'esempio di un atleta che subisce i colpi e vince, similmente il vescovo deve stare fermo e sopportare ogni cosa per Dio e per i fedeli. Nel IV capitolo Ignazio suggerisce di preoccuparsi delle vedove, degli schiavi e di tutti i fedeli, di avere la vera libertà. Nel V capitolo l'autore si dedica ai consigli che riguardano la vita matrimoniale e la castità. Ogni cosa deve essere fatta per la gloria di Dio. Eccezionalmente il VI capitolo è indirizzato ai fedeli. Ignazio chiama tutti a riunirsi intorno al vescovo. Nel VII capitolo, come nelle due ultime lettere, Ignazio, avendo ricevuto buone notizie dalla propria Chiesa, del ritorno della pace, chiede Policarpo di eleggere uno, che porti in Siria gli auguri dalla Chiesa di Smirne. Il cristiano deve vivere non per sé, ma per servire al Dio. L'ultimo, VIII capitolo, contiene il congedo.

25 Ibidem, 12, 2, SCh 10, 142: „Saluto il vescovo degno di Dio, il venerabile presbiterato, i diaconi miei conservi e uno ad uno tutti insieme nel nome di Gesù Cristo, nella sua carne e nel suo sangue, nella passione e nella resurrezione corporale e spirituale in unione a Dio e a voi". 
Nella lettera a Policarpo Ignazio una volta sola parla direttamente dei presbiteri, nel capitolo VI che, come unico, è indirizzato alla comunità.

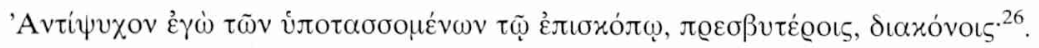

Ignazio ancora una volta ricorda di sottomettersi al vescovo, ai presbiteri e ai diaconi. Afferma la propria opinione, offrendo la sua vita per quelli che fanno così. La struttura della Chiesa particolare è ben esposta. Il capo è il vescovo. I presbiteri e i diaconi sono suoi collaboratori, ma hanno impegni diversi. Noi ci concentriamo sui presbiteri, che formano il collegio presbiterale, un gruppo di consiglieri che sono più vicini al vescovo. Il loro impegno pastorale deriva dal vescovo, ma servono il vescovo come quelli più vicini, che lo aiutano con la loro saggezza e l'esperienza. Essi formano una parte sostanziale nella Chiesa particolare.

\section{$* * *$}

Ignazio nelle sue sette lettere 23 volte dirige il suo pensiero ai presbiteri.

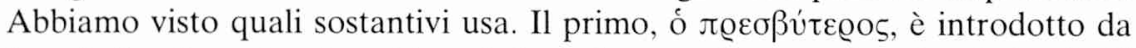
Ignazio 9 volte, nei casi genitivo, dativo ed accusativo, tutte le volte al plurale. Il

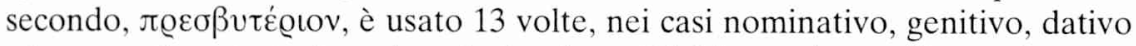

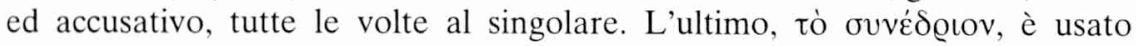
solamente una volta.

Ignazio offre una prospettiva dei presbiteri di tipo descrittivo. La sua visione è ecclesiale, ma soprattutto pratica, utile. La missione del presbitero ha la sua sorgente in $\mathrm{Cristo}^{27}$. I presbiteri, come il vescovo, devono avere il pensiero di Cristo ${ }^{28}$. Il presbitero compie la funzione del mediatore fra Dio e gli uomini2 $^{29}$. Ignazio consiglia l'unità dei presbiteri con il vescovo non per sottolineare la potestà gerarchica, ma per ricordare che l'atmosfera ecclesiale si alimenta di armonia e di comunione ${ }^{30}$. La vicinanza tra il vescovo, i presbiteri ed i fedeli è soprannaturale ${ }^{31}$. L'unità del pensiero del vescovo e dei presbiteri attua concordemente la volontà di $\mathrm{Dio}^{32}$. Il collegio dei presbiteri è immagine

${ }^{26}$ Epistula ad Polycarpum, 6, 1, SCh 10, 150: „Offro in cambio la vita per quelli che sono sottomessi al vescovo, ai presbiteri e ai diaconi e con loro vorrei essere partecipe in Dio".

27 Cf. P. Meloni, La missione del vescovo in Ignazio di Antiochia e nella liturgia, in: S. Felici (ed.), La formazione al sacerdozio ministeriale nella catechesi e nella testimonianza di vita dei Padri [= Biblioteca di Scienze Religiose 98], Roma 1992, 22.

${ }^{28}$ Cf. ibidem, 23.
${ }^{29}$ Cf. ibidem, 24.
${ }^{30}$ Cf. ibidem, 27.
${ }^{31}$ Cf. ibidem, 29.
${ }^{32}$ Cf. ibidem, 30. 
del collegio degli apostoli ${ }^{33}$. Ai presbiteri Ignazio raccomanda anche le virtù proposte a tutti i credenti: la saldezza nella fede, l'umiltà, lo spirito di pace, la bontà, la purezza, la temperanza, il rispetto, la sapienza, la serenità, la fuga dall'ira, l'impegno nella vita sociale, il dono di sé, la vittoria sulla gelosia, la magnanimità, la fortezza e la perseveranza sino alla fine, l'equità, la calma, la pazienza, la semplicità, la prudenza, lo zelo, la fede perseverante nella preghiera e nell'annunzio del Vangelo ${ }^{34}$. Il presbiterio deve essere in armonia con il vescovo e con il popolo ${ }^{35}$.

Ignazio presenta l'immagine del presbitero, nascosta nelle sue lettere. Accanto alle sue caratteristiche, espone nella struttura della Chiesa il posto particolare dei presbiteri. Anzitutto sottolinea l'unità che collega i presbiteri con il vescovo ed i fedeli. Con grazie a Ignazio, possiamo meglio scoprire chi erano i presbiteri nel II secolo della Chiesa.

\section{QUID S. IGNATIUS ANTIOCHENUS IN EPISTULIS SUIS DE PRESBYTERIS PUTAVERIT?}

(Argumentum)

Studium de presbytero apud Ignatium Antiochenum tractat: de eius persona, de eius navitate ecclesiastica, de eius magisterio. Post introductionem de vita, origine, martyrio et epistulis inquisivimus, quibus definitionibus utatur Ignatius scribendo de presbytero. Deinde investigamus explicationem singulorum epistularum fragmentorum. In fine, in compendio studia et praxim Ignatii exhibere conati sumus.

\footnotetext{
${ }^{33}$ Cf. ibidem.

${ }^{34}$ Cf. ibidem, 33.

${ }^{35}$ Cf. C. Riggi, Il sacerdozio ministeriale nel pensiero di Ignazio di Antiochia, in: S. Felici (ed.), op. cit., 45.
} 